\title{
Haben die Konzentrationen von Interleukin 6, Procalcitonin und CRP bei Intensivpatienten während des ersten Fieberanstieges eine prognostische Bedeutung?
}

\author{
Interleukin 6, procalcitonin and CRP levels during first increase of fever in \\ intensive care patients
}

\author{
Peter Fraunberger ${ }^{1, *}$, Ernst Holler ${ }^{2}$, Klaus G. \\ Parhofer $^{3}$, Autar K. Walli ${ }^{4}$ und Dietrich Seidel ${ }^{4}$ \\ ${ }^{1}$ Medizinisches Zentrallabor, Feldkirch, Österreich \\ ${ }^{2}$ Abteilung für Hämatologie und Onkologie, Universität \\ Regensburg, Deutschland \\ ${ }^{3}$ II. Medizinische Klinik, Klinikum Großhadern, \\ Universität München, Deutschland \\ ${ }^{4}$ Institut für Klinische Chemie, Klinikum Großhadern, \\ Universität München, Deutschland
}

\section{Zusammenfassung}

Zur Untersuchung der klinischen Bedeutung von Biomarkern während intensivmedizinischer Betreuung wurden Interleukin-6 (IL-6), Procalcitonin (PCT) and Creaktives Protein (CRP) bei 38 kritisch kranken Patienten während des ersten Fieberanstieges über $38,3^{\circ} \mathrm{C}$ gemessen. Bereits zum Zeitpunkt des Fieberanstieges konnten signifikant höhere IL-6-Konzentrationen bei Patienten verzeichnet werden, welche die Erkrankung nicht überlebten. Insbesondere IL-6-Werte >1000 pg/mL waren mit sehr hoher Mortalität assoziiert. Sensitivität, Spezifität, positive and negative Vorhersagewerte zur Beurteilung der Mortalität waren für IL-6 höher als für PCT und CRP. ROC-Analysen zeigten die höchste prognostische Wertigkeit für IL-6-Konzentrationen im Vergleich zu PCT und CRP-Konzentrationen. Diese Daten unterstützen die Hypothese, dass eine deutlich erhöhte IL-6-Konzentration bereits zum Zeitpunkt des ersten Fieberanstieges ein guter Frühmarker zur Erkennung von Hochrisikopatienten mit hoher Mortalität ist.

Schlüsselwörter: Fieber; Infektion; Procalcitonin; Prognose; Sepsis; Zytokine.

\footnotetext{
Abstract

Serum levels of interleukin 6 (IL-6), procalcitonin (PCT), and $\mathrm{C}$-reactive protein (CRP) were measured in 38 criti-

*Korrespondenz: Priv.-Doz. Dr. med. Peter Fraunberger, Medizinisches Zentrallaboratorium $\mathrm{GmbH}$, Carinagasse 41, A-6800 Feldkirch, Osterreich Tel.: + 435522 3480-0 E-Mail: p.fraunberger@mzl.at
}

cally ill patients immediately after an increase in body temperature above $38.3^{\circ} \mathrm{C}$. Ten healthy controls were also included for comparison. The onset of fever was accompanied by elevated circulating levels of all the 3 markers in comparison to healthy control subjects. However, only IL-6 levels were significantly higher $(p<0.05)$ in nonsurvivors compared with survivors. Sensitivity, specificity, positive, and negative predictive values for survival were higher for IL-6 in comparison to levels of PCT and CRP. Areas under receiver characteristic operating curves revealed the highest area under the curve for IL-6 compared to PCT and CRP. These data suggest that IL-6 rather than PCT or CRP may be an early predictor of prognosis and mortality in patients with the onset of fever.

Keywords: cytokines; fever; infection; procalcitonin; prognosis; sepsis.

\section{Einleitung}

Fieber $\left(>38,3^{\circ} \mathrm{C}\right)$ stellt einen der ersten Indikatoren einer beginnenden Komplikation bei kritisch kranken Intensivpatienten dar und ist mit erhöhter Mortalität assoziiert [1, 2]. Zum Zeitpunkt des ersten Fieberanstieges ist allerdings häufig noch unklar, ob es sich um eine unkritische Temperaturerhöhung oder um den Beginn einer schweren infektiösen Komplikation handelt. Insbesondere bei neutropenische Patienten ist der Fieberanstieg mit einem hohen Risiko für die Entstehung einer Sepsis assoziiert und hat in der Regel eine intensive antibiotische Therapie zur Folge. Zum Zeitpunkt des Fieberanstieges stellt sich daher häufig die Frage nach dem Risiko der Entwicklung einer Sepsis, um einerseits frühzeitig anti-infektiv zu behandeln und andererseits eine Übertherapie zu vermeiden. Ziel der vorliegenden Studie war die Prüfung, ob messbare Biomarker geeignet sind, das Risiko einer Sepsis früh abzuschätzen.

Von zahlreichen Labormarkern gehört das C-reaktive Protein (CRP) zu den am häufigsten gemessenen Entzündungsmarkern. Insbesondere für die Fragestellung einer Infektion oder Sepsis sind Sensitivität und Spezifität 
von CRP jedoch gering und damit zur frühen Identifizierung von Hochrisikopatienten ungeeignet. Zahlreiche Studien weisen darauf hin, dass neuere Marker wie Interleukin-6 (IL-6)- und Procalcitonin (PCT) ebenfalls erhöhte Konzentrationen bei entzündlichen Erkrankungen aufweisen und besser zur Diagnose einer Sepsis geeignet sind [3-7]. Während PCT-bakterielle Infektionen, SIRS oder Sepsis mit einer höheren Spezifität als CRP anzeigt [3, 4], weisen IL-6-Konzentrationen einen sehr frühen Anstieg auf, der deutlich vor den klinischen Zeichen einer Entzündung und auch vor dem CRP-Anstieg auftritt [5, 6] Bei Patienten mit gesicherten Sepsen konnten sowohl für erhöhte IL-6- als auch für PCT-Konzentrationen eine Korrelation mit der Mortalität gezeigt werden [8].

Zur Evaluierung von IL-6- und PCT-Konzentrationen zur frühen Erkennung von Hochrisikopatienten auf Intensivstationen im Vergleich zu CRP wurden in der vorliegenden Studie die diese drei Biomarker bei 38 Intensivpatienten prospektiv zum Zeitpunkt des Fieberanstieges gemessen und die Zusammenhänge mit der Mortalität analysiert.

\section{Material und Methoden}

\section{Patienten}

38 Intensivpatienten wurden prospektiv in die Studie eingeschlossen sobald ein Fieberanstieg nachweisbar war. Das mediane Alter der Patienten (22 Männer, 16 Frauen) betrug 56 Jahre (19-85 Jahre). Die Grunderkrankungen waren: Lymphom/Leukämie $(n=16)$, solider Tumor $(n=2)$, Leberzirrhose $(n=8)$, akute oder chronische Herzerkrankung $(n=10)$, Diabetes mellitus $(n=2)$, Niereninsuffizienz $(n=3)$, Magenulkus $(n=3)$, Dermatomyositis $(n=1)$, Mesenterialinfarkt $(n=1)$, Drogenabhängigkeit $(n=1)$ sowie Lungenembolie $(n=1)$.

Die Körpertemperatur wurde täglich dreimal gemessen. Fieber wurde definiert als Anstieg der Körpertemperatur auf $>38,3^{\circ} \mathrm{C}$. Anschließend wurden die Krankenhausmortalität, die Grunderkrankung und der klinische Verlauf der Patienten bezüglich der Entstehung einer Sepsis, Pneumonie oder nicht-infektiöser Komplikationen dokumentiert. Sepsis wurde entsprechend Bone-Kriterien definiert [9]. Pneumonie wurde diagnostiziert entsprechend den Richtlinien von Maschmeyer et al. [10]. Zum Vergleich wurden 10 gesunde Kontrollen eingeschlossen.

\section{Blutentnahme und Analyse}

Sobald ein Temperaturanstieg auf $38,3^{\circ} \mathrm{C}$ gemessen wurde, erfolgte die venöse Blutentnahme. Das Blut wurde zentrifugiert und Serum in Aliquots bis zur Analyse bei $-70^{\circ} \mathrm{C}$ aufbewahrt. CRP wurde an einem Hitachi 911 (Roche, Mannheim, Deutschland) turbidimetrisch gemessen. IL-6 wurde mittels eines automatisierten ELISA am CobasCore $^{\circledR}$ (Roche, Mannheim, Deutschland) gemessen. Die Messung der PCT-Spiegel erfolgte mit einem immunoluminometrischen Test (LUMltest, Fa. Brahms Diagnostica, Berlin, Deutschland).

\section{Statistische Analyse}

Die Serumkonzentrationen der Biomarker sind angegeben als Median \pm Standardabweichung. Der statistische Vergleich zwischen überlebenden und verstorbenen Patienten erfolgte mit Hilfe des Mann-Whitney-U-Testes. Ein $p$-Wert kleiner 0,05 wurde als statistisch signifikant angesehen. Die prädiktive Bedeutung der Biomarker wurde mittels ROC-(receiver operating characteristic)Analyse als Plot der Sensitivität des jeweiligen Testes gegen die falsch-positive Rate für alle möglichen Cut-off Level ermittelt. Die diagnostische Aussagekraft von IL-6, PCT and CRP wurde als AUC (area under the ROC curve) mit dem 95\%-Konfidenzintervall ausgedrückt. Der $\chi^{2}-$ Test wurde für die Berechnung von Sensitivität, Spezifität, negativem und positiven prädiktiven Werten für die medianen ersten und dritten Quartile der Daten für alle Patienten dargestellt.

\section{Ergebnisse}

Im weiteren Verlauf nach dem ersten Fieberanstieg entwickelten 10 Patienten eine Sepsis und 8 Patienten eine beidseitige Pneumonie. Die verbleibenden Patienten hatten entweder lokale Infektionen $(n=6)$, Mokardinfarkt $(n=2)$, Knochenmarkabstoßung $(n=1)$ oder Leberversagen $(n=1)$ als Fieberursache. Von den 38 Patienten verstarben 21 (55\%) im Laufe des Krankenhausaufenthaltes.

Der Fieberanstieg war verbunden mit einem signifikanten Anstieg der Serumkonzentrationen aller drei Biomar-

Tabelle 1 IL-6-, PCT- und CRP-Konzentrationen bei intensivpflichtigen Patienten zum Zeitpunkt des Fieberanstieges $\left(>38,3^{\circ} \mathrm{C}\right)$.

\begin{tabular}{llll}
\hline & IL-6 [pg/mL] & PCT $[\mathrm{ng} / \mathrm{mL}]$ & $\mathrm{CRP}[\mathrm{mg} / \mathrm{dL}]$ \\
\hline Überlebt $(\mathrm{n}=17)$ & $697 \pm 432$ & $27,9 \pm 25,9$ & $13,4 \pm 4,5$ \\
Verstorben $(\mathrm{n}=21)$ & $1706 \pm 420$ & $59,7 \pm 48,7$ & $15,4 \pm 4,2$ \\
P (Kruskal-Wallis-test) & 0,001 & 0,091 & 0,607 \\
Sensitivität* & $71,4 \%$ & $57,1 \%$ & $57,1 \%$ \\
Spezifität* $^{*}$ & $76,5 \%$ & $58,8 \%$ & $58,8 \%$ \\
Pos. prädiktiver Wert* & $78,9 \%$ & $63,2 \%$ & $63,2 \%$ \\
Neg. prädiktiver Wert* & $68,4 \%$ & $52,6 \%$ & $52,6 \%$ \\
\hline
\end{tabular}

Mittelwert $\pm 95 \%$-Konfidenzintervall.

*berechnet mit dem Median aller Patienten als Grenzwert. 


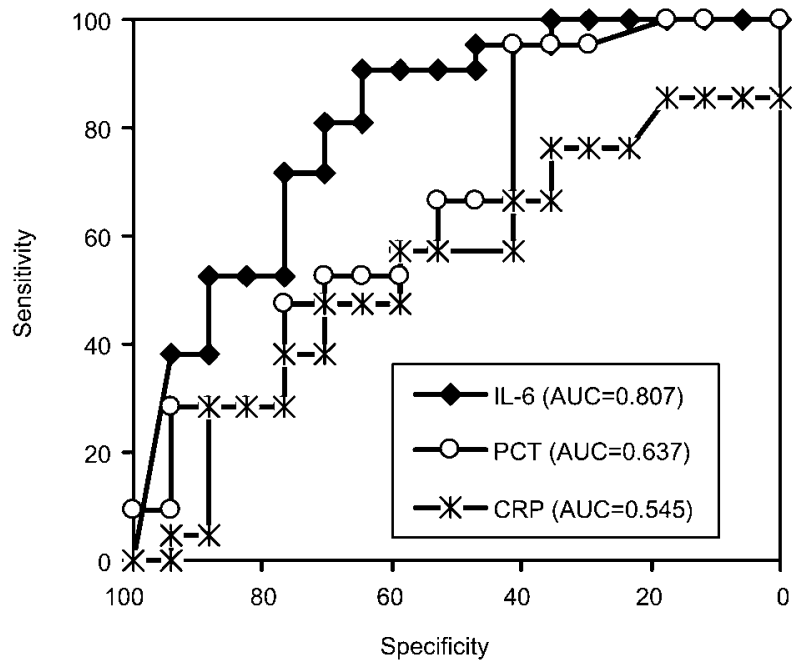

Abbildung 1: ROC-Analyse für das Überleben für IL-6-, PCTund CRP-Konzentrationen zum Zeitpunkt des Fieberanstieges.

ker (IL-6: 1000 (44-2815) pg/mL; PCT: 6,3 (0,3$510,7) \mathrm{ng} / \mathrm{mL}$; CRP 11,4 (0,6-35,0) mg/dL) im Vergleich zu den gesunden Normalpersonen (IL-6: <10 pg/mL; PCT: $<1 \mathrm{ng} / \mathrm{mL}$; CRP $<0,5 \mathrm{mg} / \mathrm{dL}$ ). Allerdings waren nur die IL-6-Konzentrationen signifikant $(p<0,05)$ höher bei den Patienten die verstarben, im Vergleich zu den überlebenden Patienten. PCT- und CRP-Konzentrationen unterschieden sich nicht signifikant zwischen diesen beiden Patientengruppen. Auch die Sensitivität, Spezifität sowie die negativen und positiven prädiktiven Werte waren für IL-6 höher im Vergleich zu PCT and CRP (Tabelle 1).

Um die diagnostische Wertigkeit von IL-6, PCT und CRP zu vergleichen, wurden mittels ROC-Analyse die AUC (area under the curve) berechnet (Abbildung 1). Die AUC der IL-6-Spiegel war mit 0,807 (Konfidenzintervall 0,668-0,951) höher als die AUC der PCT- $(0,637$, Konfidenzintervall 0,483-0,839) und CRP-Konzentrationen $(0,545$, Konfidenzintervall 0,362-0,736).

\section{Diskussion}

Die Freisetzung proinflammatorischer Zytokine wie IL-1, IL-6 and TNF stellt einen zentralen Mechanismus für die Entstehung und den Verlauf einer Entzündungsreaktion dar. In experimentellen In-vivo-Untersuchungen konnte gezeigt werden, dass bereits wenige Minuten nach Auslösung der Entzündungsreaktion TNF und IL-1 sowie nachfolgend IL-6 freigesetzt werden. Alle drei Zytokine spielen eine zentrale Rolle bei der Auslösung der AkutePhase-Reaktion mit dem Auftreten von Fieber [12]. Während TNF und IL-1 jedoch häufig nur pulsatil freigesetzt werden, ist IL-6 wegen der längeren biologischen Halbwertzeit länger in der Zirkulation nachweisbar. Darüber hinaus konnte ein Zusammenhang zwischen der IL-6Konzentration und der Prognose nachgewiesen werden [5-7]. In der vorliegenden Studie konnte diese prognostische Bedeutung bestätigt werden. Zum Zeitpunkt des
Fieberanstieges waren insbesondere IL-6-Konzentrationen über 1000 pg/mL mit sehr hoher Mortalität assoziiert.

Im Gegensatz zu den proinflammatorischen Zytokinen ist über die pathophysiologische Bedeutung von PCT wenig bekannt. Erhöhte PCT-Konzentrationen konnten bei einer Vielzahl von entzündlichen Erkrankungen, wie bakteriellen Infektionen und Sepsis nachgewiesen werden. Insbesondere bei Patienten mit schweren entzündlichen Erkrankungen konnte darüber hinaus eine Korrelation der PCT-Konzentrationen mit der Schwere der Erkrankung aufgezeigt werden [3, 4, 12]. In der vorliegenden Studie erwiesen sich allerdings zum Zeitpunkt des Fieberanstieges IL-6-Konzentrationen als bessere Prognosemarker im Vergleich zu PCT und CRP. Ursache hierfür könnten die unterschiedlichen Zeitverläufe der IL6-, PCT- und CRP-Freisetzung sein. In-vivo-Studien haben gezeigt, dass bereits wenige Minuten nach Endotoxin-Gabe IL-6 in der Zirkulation nachweisbar ist, wogegen PCT erst nach 2 Stunden und CRP erst nach 6 Stunden im Serum ansteigt [14]. Obwohl in der vorliegenden Studie keine Verlaufsuntersuchungen durchgeführt wurden, konnte in früheren Untersuchungen [15] gezeigt werden, dass IL-6-Konzentrationen zum Zeitpunkt des Fieberanstieges einen Gipfel aufweisen und anschließend wieder abnehmen. PCT steigt etwas später im Serum an, ist dann jedoch auf Grund der längeren biologischen Halbwertzeit deutlich länger nachweisbar als IL-6. Das könnte erklären, warum zum frühen Zeitpunkt des Fieberanstieges IL-6 der bessere Prognosemarker ist, wogegen PCT bei Patienten mit etablierter Sepsis einen besseren Marker des Schweregrades darstellt [16].

Zusammengefasst konnte in der vorliegenden Studie gezeigt werden, dass IL-6 bereits zu dem frühen Zeitpunkt des Fieberanstieges ein geeigneter Marker zur Erkennung von Risikopatienten darstellt. Weitere prospektive Studien sind notwendig um zu klären, ob die frühe IL-6-Messung geeignet ist, durch frühere, gezielte anti-infektive Therapie die Prognose von Intensivpatienten mit Fieberanstieg zu verbessern.

\section{Literatur}

1. O'Grady NP, Barie PS, Bartlett J, Bleck T, Garvey G, Jacobi $J$, et al. Practice parameters for evaluating new fever in critically ill adult patients. Task Force of the American College of Critical Care Medicine of the Society of Critical Care Medicine in collaboration with the Infectious Disease Society of America. Crit Care Med 1998;26:392-408.

2. Circiumaru B, Baldock G, Cohen J. A prospective study of fever in the intensive care unit. Intensive Care Med 1999; 25:668-73.

3. Simon L, Gauvin F, Amre DK, Saint-Louis P, Lacroix J. Serum procalcitonin and C-reactive protein levels as markers of bacterial infection: a systematic review and meta-analysis. Clin Infect Dis 2004;39:206-17.

4. Harbarth S, Holeckova K, Froidevaux C, Pittet D, Ricou B, Grau GE, et al. Diagnostic value of procalcitonin, interleukin6 , and interleukin-8 in critically ill patients admitted with sus- 
pected sepsis. Am J Respir Crit Care Med 2001;164:396402.

5. Steinmetz HT, Herbertz A, Bertram M, Diehl V. Increase in interleukin-6 serum level preceding fever in granulocytopenia and correlation with death from sepsis. J Infect Dis 1995;171:225-8.

6. Le Moine O, Deviere J, Devaster JM, Crusiaux A, Durand F, Bernuau J, et al. Interleukin-6: an early marker of bacterial infection in decompensated cirrhosis. J Hepatol 1994;20: 819-24.

7. Rosenbloom AJ, Pinsky MR, Bryant JL, Shin A, Tran T, Whiteside T. Leukocyte activation in the peripheral blood of patients with cirrhosis of the liver and SIRS. Correlation with serum interleukin-6 levels and organ dysfunction. J Am Med Assoc 1995;274:58-65.

8. Wunder C, Eichelbrönner O, Roewer N. Are IL-6, IL-10 and PCT plasma concentrations reliable for outcome prediction in severe sepsis? A comparison with APACHE III and SAPS II. Inflamm Res 2004;53:158-63.

9. Bone RC, Balk RA, Cerra FB, Dellinger RP, Fein AM, Knaus $W A$, et al. Definitions for sepsis and organ failure and guidelines for the use of innovative therapies in sepsis. The ACCP/SCCM Consensus Conference Committee. American College of Chest Physicians/Society of Critical Care Medicine. Chest 1992;101:1644-55.

10. Maschmeyer G, Beinert T, Buchheidt D, Einsele H, Heussel $\mathrm{CP}$, Kiehl M, et al. Infectious Diseases Working Party (AGI-
$\mathrm{HO}$ ) of the German Society of Hematology and Oncology (DGHO). Diagnosis and antimicrobial therapy of pulmonary infiltrates in febrile neutropenic patients - guidelines of the Infectious Diseases Working Party (AGIHO) of the German Society of Hematology and Oncology (DGHO). Ann Hematol 2003;82:118-26.

11. Fraunberger $P$, Pfeiffer $M$, Cremer $P$, Holler E, Nagel D, Dehart I, et al. Validation of an automated enzyme immunoassay for Interleukin- 6 for routine clinical use. Clin Chem Lab Med 1998;36:797-801.

12. Zetterström $M$, Sundgren-Andersson $A K$, Ostlund $P$, Bartfai $\mathrm{T}$. Delineation of the proinflammatory cytokine cascade in fever induction. Ann N Y Acad Sci 1998;856:48-52.

13. de Werra I, Jaccard C, Corradin SB, Chiolero R, Yersin B, Gallati $\mathrm{H}$, et al. Cytokines, nitrite/nitrate, soluble tumor necrosis factor receptors, and procalcitonin concentrations: comparisons in patients with septic shock, cardiogenic shock, and bacterial pneumonia. Crit Care Med 1997;25: 607-13.

14. Dandona P, Nix D, Wilson MF, Aljada A, Love J, Assicot M, et al. Procalcitonin increase after endotoxin injection in normal subjects. J Clin Endocrinol Metab 1994;79:1605-8.

15. Groeneveld AB, Tacx AN, Bossink AW, van Mierlo GJ, Hack CE. Circulating inflammatory mediators predict shock and mortality in febrile patients with microbial infection. Clin Immunol 2003;106:106-15.

16. Carrol ED, Thomson AP, Hart CA. Procalcitonin as a marker of sepsis. Int J Antimicrob Agents 2002;20:1-9. 